\title{
HIDROQUÍMICA DE UN RÍO DE CARACTERÍSTICAS SEMIÁRIDAS (RÍO CHICAMO; MURCIA). ANÁLISIS ESPACIO-TEMPORAL
}

\author{
M.R. Vidal-Abarca; M.L. Suárez; J.L. Moreno; R. Gómez; I. Sánchez \\ Departamento de Ecologia e Hidrologia. Universidad de Murcia. Campus de Espinardo 30100. MURCIA.
}

\begin{abstract}
RESUMEN
En este estudio se describen y analizan las variaciones espacio-temporales de 20 parámetros físicos y químicos medidos en un sector de cauce del tramo medio del Rio Chicamo, un n o salino, temporal y espacialmente intermitente, localizado en el sureste ibérico. En general, el Rio Chicamo presenta aguas hiposalinas, bien oxigenadas, alcalinas y muy ricas en nutrientes. La elevada concentracidn de nitrogeno en el agua, sobretodo en forma de nitratos $(2.2 \mathrm{mg} / \mathrm{L})$, es consecuencia de los materiales margosos de origen sedimentario de la cuenca de drenaje. Las caracteristicas fisonómicas de las estaciones de muestreo y la dinámica hidrológica compleja, que incluye varias fases a lo largo del año, parecen explicar la evolucidn espacial y temporal de muchas de las variables fisicas y quirnicas analizadas. La combinación de un análisis de ordenacidn (PCA) con otro de clasificacidn (cluster) sobre una matriz de datos previamente seleccionados, permite diferenciar distintas fases hidrológicas caracterizadas por una combinación de variables fisicas y quimicas (anchura de la lámina de agua, caudal, conductividad, saturación de oxigeno y nutrientes)
\end{abstract}

Palabras clave: río salino, rio temporal, caracteristicas fisicas y quimicas, dinámica hidrologica, sureste ibérico

\begin{abstract}
Spatio-temporal variations of 20 physical and chemical parameters were measured in the midle sector of the Chicamo stream. The Chicamo is a saline, temporal, and spatially intermittent stream located in Murcia (southeast Spain). The water is hyposaline, with high dissolved oxygen and alkalinity, and it is rich in nutrients. The high values of nitrate are due to sedimentary origin of marls in the watershed. The characteristicfeatures of the samplig sites and the complex hydrological dynamic of Chicamo stream may explain the water hydrochemical variability. Multivariate techniques of ordination and classification were applied to a matrix with selected physical and chemical parameters which allowed us to define three hydrological phases, each characterized by differentfeatures (saturated channel width, discharge, conductivity, saturation oxygen and nutrients)
\end{abstract}

Keywords: saline stream, temporal stream, physical and chemical characteristics, hydrological dynamics, southeast Iberian peninsula.

\section{INTRODUCCIÓN}

Aún son escasos los estudios que analizan las caracteristicas hidroquimicas de los ecosistemas acutiticos continentales con dinámicas hidrológicas complejas, como ramblas, rios temporales y/o rios espacialmente intermitentes, sometidos a perturbaciones hidrológicas naturales de distinta magnitud (riadas, sequias, etc). Cabe destacar, no obstante, los trabajos en Sycamore Creek, en el desierto de Sonora (Arizona), sobre la dinámica hidroquimica tras las avenidas de agua (Fisher \& Minkley, 1978; Fisher et al., 1982), o durante las sequias (Stanley \& Fisher, 1992), e incluso el papel que juega el hiporreos como un componente más del ecosistema fluvial (Valett et al., 1990; Stanley \& Boulton, 1995). De igual manera, resultan relevantes los trabajos de Boulton y Suter (1986) y Boulton y Lake (1990), sobre rios temporales e intermitentes australianos, o los de 
arroyos temporales de las praderas americanas (Matthews, 1988; Zale et al., 1989; Dodds, et al., 1996).

En Espaiia cabe destacar, el estudio del arroyo temporal de la Montesina (Sierra Morena) (Maltchick \& Mollá, 1994; Maltchick et al.,1994; 1998; Moll6 et al., 1994), los realizados en rios temporales de Cataluiia (Sabater \& Armengol, 1986; Sabater et al., 1991; 1992; Marti \& Sabater, 1996) y los enmarcados en ambientes áridos del Sureste ibérico, como los trabajos de Ortega et al., (1988) en una rambla sometida a intensas avenidas de agua, los de Moreno et al., (1995) en ramblas del litoral murciano de temporalidad extrema y los de Gomez (1995) en humedales asociados a ramblas de carácter salino y temporal.
De forma general, en estos trabajos se marca la temporalidad como el carácter que mejor explica la variación de los parámetros físicos, químicos y/o biológicos analizados (Comin \& Williams, 1994). De hecho a partir de ellos, algunos autores han planteado hipótesis y modelos de funcionamiento para este tipo de ecosistemas, en base a las distintas fases hidrológicas que es posible definir. Asi, Boulton \& Lake (1990), en un estudio bianual realizado en dos rios intermitentes australianos definen cinco fases hidrológicas (pre-flujo, flujo temprano, flujo principal, disminución del flujo y post-flujo) cada una de ellas caracterizada por una combinación compleja de distintas variables fisicas y quimicas. De manera similar, Stanley \& Fisher (1992) describen un

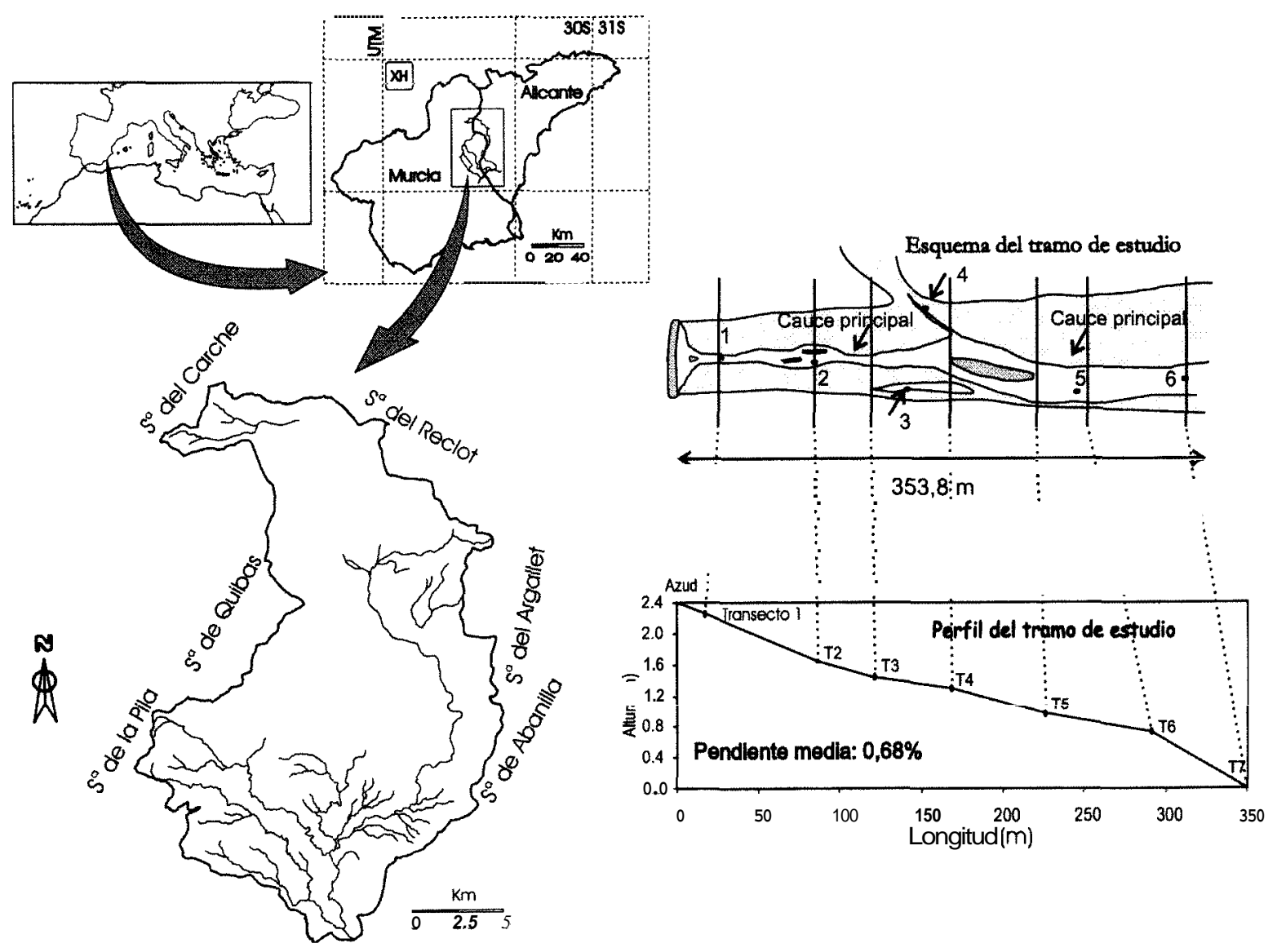

Figura 1. Localizacidn geográfica del Rio Chicamo, situacidn de las estaciones de muestreo y pendiente y transectos en el tramo de estudio. Location of the Chicamo stream, the sampling sites, the transects in the reach studied and slope. 
marco conceptual basado en la definición y caracterización de las tres fases hidrológicas, que se observan durante un proceso de sequia (Fase I: pérdida progresiva del flujo de agua; Fase II: sequia o ausencia de agua superficial; Fase 111: restitución del flujo de agua), en función de los cambios detectados para distintas variables biológicas, interpretados en términos de la teoria de la estabilidad en relación con la resistencia y resiliencia de las comunidades.

Mas recientemente, se ha prestado atención a la diferencia de comportamiento de las zonas de corriente ("runs" o "riffles") frente a las pozas ("pools") en el proceso de sequia de un tramo de cauce. De hecho, Stanley et al. (1997), han propuesto un esquema de funcionamiento para los rios intermitentes, basado en los distintos modelos de sequia que sigue cada tipo de habitat ("runs", "riffles" y "pools") definidos a una escala de tramo. Estos autores muestran como los rios son espacialmente dinhmicos, según ciclos de expansión, contracción y fragmentación.

Finalmente, la incorporación de los efectos de las perturbaciones a distintas escalas espaciales y temporales, y las relaciones y efectos que se producen entre los distintos compartimentos que caracterizan a un ecosistema fluvial, ha dado lugar a la formulacion de una nueva teoria: "A Telescoping Ecosystem Model": (Fisher et al., 1998a) aún por probar.

En vista a estos trabajos resulta interesante analizar si estos modelos pueden ser aplicados a rios de caracteristicas ambientales similares como el Rio Chicamo, un afluente del Rio Segura, situado en el sureste ibérico, en un área ambientalmente arida. Asi, en este estudio se pretende, en primer lugar, analizar de forma conjunta la variación espacial y temporal de 20 parámetros fisicos y quimicos, medidos durante un ciclo hidrológico anual (1994) en seis estaciones de muestreo. En segundo lugar, analizar qué factores ambientales pueden explicar estas variaciones espacio-temporales y finalmente discutir sobre la idoniedad y utilidad de los modelos anteriores en el esquema general de variabilidad e irregularidad que caracteriza a estos ecosistemas.

\section{EL AREA DE ESTUDIO}

El Rio Chicamo es un afluente del Rio Segura, por su márgen izquierda (Fig. 1). Su longitud es de $59.4 \mathrm{~km}$ aunque sólo unos $22 \mathrm{~km}$ mantienen agua de forma temporal o permanente. Ello es debido tanto a las condiciones climaticas del área como a las intensas extracciones de agua que se realizan desde su nacimiento, para abastecer a la agricultura de regadio.

La cuenca del Rio Chicamo drena una superficie de $502 \mathrm{~km}^{2}$ y se localiza en el sector más árido de la Región de Murcia, con temperaturas medias anuales de $18^{\circ} \mathrm{C}$ y una precipitación media anual de $250 \mathrm{~mm}$ (Vidal-Abarca et al., 1987). Los materiales geológicos son margas grises, ricas en yeso, blandas y facilmente erosionables, que organizan los paisajes de bad-lands, tipicos de muchos sectores del sureste ibérico.

El estudio se llevó a cabo en un sector del tramo medio del Rio Chicamo de $350 \mathrm{~m}$ de longitud, donde el agua fluye de forma permanente durante todo el ciclo hidrológico anual. Durante el periodo de estudio (desde enero hasta diciembre de 1994) la temperatura media ambiental fue de $19^{\circ} \mathrm{C}$ y la precipitación anual no super6 los $150 \mathrm{~mm}$ (Fig. 2). El inicio del tramo de estudio comprende un pequeiio azud, hoy dia totalmente colmatado y, en consecuencia muy naturalizado, bajo el cual se produce una descarga de agua subterranea, origen del agua del tramo de estudio. Unicamente cuando se producen aguaceros de cierta intensidad circula agua por encima del azud.

El sustrato del cauce está constituido por margas impermeables sobre las que se depositan materiales mas blandos (limos y arcilla). Existe una clara dominancia de los materiales orghnicos, predominando la fracción fina de la materia orgánica bentónica (FPOM) debido a la descomposición de los productores primarios (Martinez, et $a l, 1998)$. Sobre ella, una capa casi uniforme de perifiton cubre la totalidad del cauce durante la mayor parte del año. No obstante, en las pozas crecen otros productores primarios como Chara vulgaris, mientras que en las zonas de corriente aparecen pequeiias manchas de Cludophora glomerata y Enteromorphu intestinalis. 

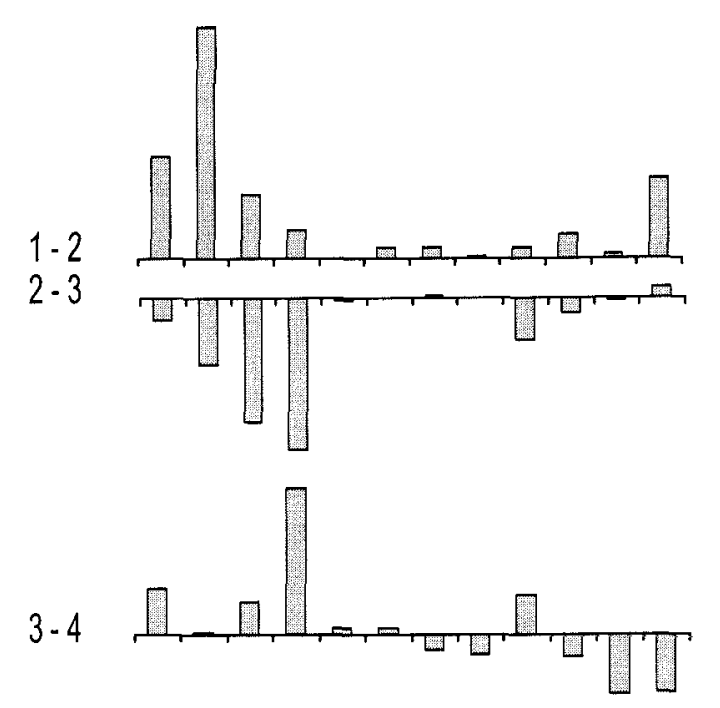

$4-5$
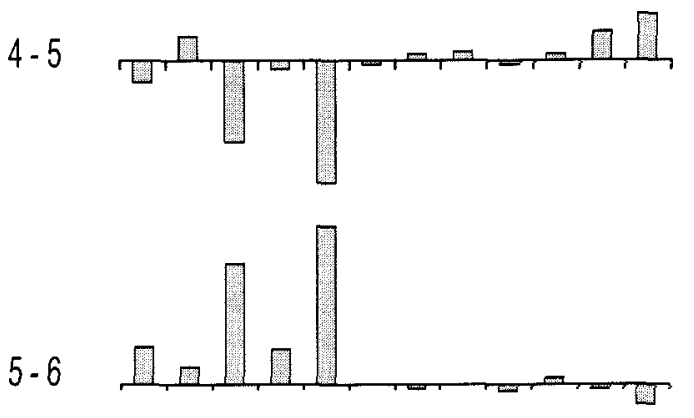

$6-7$
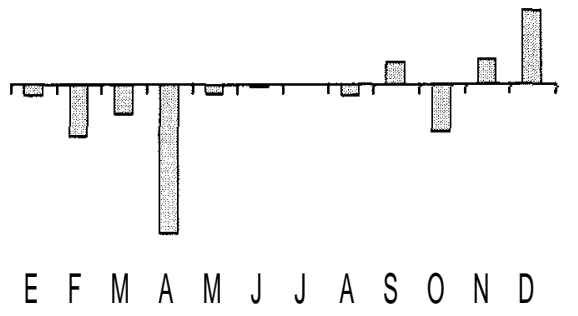

\section{Transectos}

Figura 2. Variación mensual de las diferencias de caudal, medidas en los siete transectos del tramo de estudio en el Rio Chícamo. Monthly variobility in the water flow differences, meusured in the seven studied strechts at Chicumo stream.

La vegetación ribereiia apenas existe a causa de las avenidas de agua. Unicamente y de forma muy aislada, aparecen pies jovenes de Tamarix canariensis y pequeños nucleos de Phragmites australis. Junto a ellos, algunas especies de juncos (Juncus maritimus, Scirpus holoschoenus, etc) se distribuyen a 10 largo del cauce.

\section{METODOLOGÍA}

Con objeto de recoger la maxima diversidad espacial de habitats del tramo de estudio, se seleccionaron seis estaciones de muestreo: cuatro en el cauce principal, de las cuales dos ( 1 y 6 ) se situaron en zonas de corriente y dos ( 2 y 5 ) en pozas. Otra estación, se situó en una charca lateral (3) y otra más a la salida de un pequeiio humedal que desagua al canal principal (4) (Fig. I).

Los muestreos se llevaron a cabo mensualmente durante 1994. En cada una de las estaciones se midió: la anchura, la profundidad de la lamina de agua y la velocidad de la corriente (molinete electromagnético M 201D). Asi mismo se midi6 la temperatura del agua, el $\mathrm{pH}$, la salinidad y la conductividad ( $\mathrm{pH}$-metro Crison y conductivimetro YSI-33).

Para el analisis de la composición quimica del agua se recogieron muestras, por triplicado, en botellas de polietileno (Mackereth et al., 1978) lavadas previamente con ácido, que se transportaban en nevera hasta el laboratorio y en botellas de tapón esmerilado para la medida del oxigeno disuelto segun el método de Winkler (1888). Durante una buena parte del ciclo estudiado, la recogida de agua tuvo que realizarse con jeringuillas dada la escasa profundidad de la lamina de agua.

En el Iaboratorio, Ias muestras se filtraron (filtros Whatman GF/C) para obtener los sólidos en suspensión. Una vez filtrada el agua se obtenia la medida de alcalinidad. Cuando fue necesario las muestras se congelaron, aunque los análisis de los nutrientes (N-NO;; N-NO, ; N- $\mathrm{NH}_{4}^{+} \mathrm{y}$ fósforo reactivo soluble: PRS) se realizaron antes de las 48 horas desde la toma de las muestras. Tanto para la determinación de nutrientes como para la dureza, cloruros, sulfatos, calcio, magnesio, sodio y potasio, se siguieron los tratados de A.P.H.A. (1985); Golterman et al., (1978) y Wetzel \& Likens (1991). Dado su carácter conservativo, los iones mayoritarios unicamente se midieron cuatro veces durante el ciclo hidrológico estudiado (enero, abril, julio y octubre).

Se establecieron siete transectos permanentes, transversales a la dirección del flujo de agua, con objeto de analizar en detalle las variaciones de 
caudal y la distribución de la lámina de agua superficial en el tramo de estudio.

Se utilizó un análisis de la varianza (ANOVA para test paramétricos y Kruskal-Wallis para los no parámetricos) con el fin de comprobar si existían diferencias significativas entre los valores medios mensuales de los parámetros físico-químicos estudiados. Este análisis se realizó para las cuatro estaciones localizadas en el cauce principal $(1,2,5$ y 6$)$.

Finalmente, con el fin de describir las tendencias conjuntas de variación espacial y temporal de los principales parametros fisico-quimicos analizados, se realizó un análisis de componentes principales (PCA), mediante el programa XLSTAT, versión 4.0 (Fahmy, 1999) sobre una matriz de 9 x 44. Las variables fisico-quimicas incluidas fueron: anchura de la lámina de agua, caudal, temperatura del agua, conductividad a $25^{\circ} \mathrm{C}, \mathrm{pH}$, saturación de oxigeno, N-NO; $\mathrm{N}_{-} \mathrm{NH}_{4}{ }^{+}$y $\mathrm{PRS}$, despues de eliminar el resto por aportar información redundante (salinidad, velocidad de la corriente, profundidad de la lamina de agua, oxí- geno disuelto y nitritos). De igual manera, se eliminaron de la matriz de datos las estaciones 3 y 4 dada su localización y dinamica particular, al igual que el muestreo de enero, porque no se pudo medir PRS. Todos los datos fueron transformados segun la expresión log $(x+1)$.

Finalmente, con el fin de diferenciar de forma objetiva las distintas fases hidrológicas en el cauce del Rio Chicamo, a las coordenadas de los tres primeros ejes del PCA se les aplicó un análisis de clasificacion ("cluster"), siguiendo una técnica aglomerativa con las distancias de Ward, mediante el mismo paquete estadistico (programa XLSTAT, versión 4.0).

\section{RESULTADOS}

\section{Hidrologia}

La interacción agua superficial-agua subsuperficial, en el tramo estudiado es bastante compleja (Fig. 2). En general, se detectan zonas de surgen-
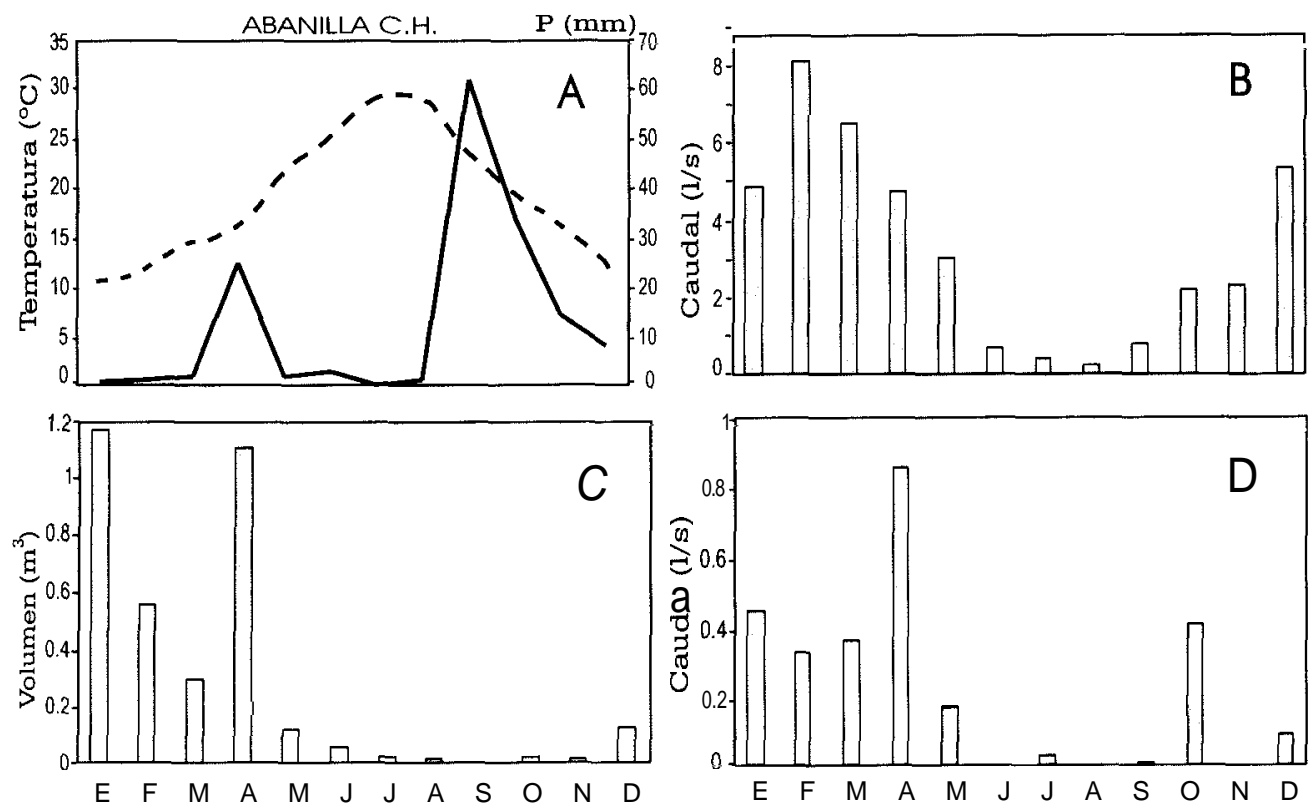

Figura 3. Climodiagrama del periodo de estudio (A) y evolución mensual del caudal medio del tramo de estudio (B), de los volúmenes de la charca lateral (estación 3) (C) y de los caudales de salida del humedal (estación 4) (D). Climatic diagram (A) and discharge of Chicamo stream during period studied (B). Evolution of capacity of lateral pool (sire 3) (C)and discharge of wetland (site 4 ) at Chicumo stream during period studied $(D)$. 
Tabla 1. Valores medios, minimos y máximos y coeficientes de variación de los parámetros fisico-quimicos medidos en las cuatro estaciones de muestreo $(1,2,5$ y 6) del cauce principal del Rio Chicamo. Mean, minimum, and maximum values and coefficients of variation of the physico-chemical parameters measured at thefour sampling sites $(I, 2,5$ y 6$)$ of the main channel in the Chicamo.

\begin{tabular}{|c|c|c|c|c|c|}
\hline & $\mathbf{n}$ & $\begin{array}{r}\text { Valor } \\
\text { medio }\end{array}$ & $\begin{array}{r}\text { Valor } \\
\text { minimo } \\
\end{array}$ & $\begin{array}{r}\text { Valor } \\
\text { maximo } \\
\end{array}$ & $\begin{array}{r}\text { C.V. } \\
\%\end{array}$ \\
\hline anchura lamina de agua (m) & 48 & 8,7 & 2,0 & 22,5 & 74,3 \\
\hline profundidad $(\mathrm{cm})$ & 48 & 7,3 & 0,7 & 32,0 & 142,1 \\
\hline velocidad corriente $(\mathrm{cm} / \mathrm{s})$ & 48 & 2,1 & 0,0 & 12,0 & 82,7 \\
\hline caudal $(1 / \mathrm{s})$ & 48 & 3,6 & 0,0 & 17,0 & 110,2 \\
\hline $\mathrm{T}^{\mathrm{a}}$ agua $(" \mathrm{C})$ & 48 & 18,6 & 7,0 & 29,5 & 31,7 \\
\hline pH & 48 & 7,8 & 7,0 & 8,3 & 4,1 \\
\hline oxigeno disuelto $(\mathrm{mg} / \mathrm{L})$ & 44 & 12,1 & 7,6 & 17,5 & 16,0 \\
\hline sólidos suspension $(\mathrm{mg} / \mathrm{L})$ & 48 & 10,2 & 0,0 & 51,1 & 112,0 \\
\hline salinidad $(\mathrm{g} / \mathrm{L})$ & 48 & 7,6 & 5,0 & 11,2 & 16,8 \\
\hline conductividad $25^{\circ} \mathrm{C}(\mathrm{mS} / \mathrm{cm})$ & 48 & 12,8 & 8,0 & 19,2 & 17,2 \\
\hline cloruros $(\mathrm{meq} / \mathrm{L})$ & 16 & 75,6 & 31,0 & 119,9 & 38,5 \\
\hline sodio (meq/L) & 16 & 88,6 & 47,3 & 120,2 & 24.5 \\
\hline potasio (meqk) & 16 & $\mathbf{0 , 3}$ & 0,1 & 0,5 & 40,3 \\
\hline calcio $(\mathrm{meq} / \mathrm{L})$ & 16 & 13,2 & 7,6 & 18,0 & 26,5 \\
\hline magnesio (meq/L) & 16 & 21,3 & 8,4 & 42,4 & 46,8 \\
\hline alcalinidad (mg/l) & 32 & 219,5 & 82,7 & 300,8 & 27,0 \\
\hline $\mathrm{N}$-nitratos $(\mathrm{mg} / \mathrm{L})$ & 48 & 2,2 & 4,3 & 5.1 & 48,3 \\
\hline $\mathrm{N}$-nitritos $(\mathrm{mg} / \mathrm{L})$ & 48 & 0,0 & inap. & 0,1 & 95,8 \\
\hline $\mathrm{N}$-amonio $(\mathrm{mg} / \mathrm{L})$ & 48 & 0,3 & 0,0 & 2,5 & 161,5 \\
\hline P-fósforo reactivo soluble $(\mu \mathrm{g} / \mathrm{L})$ & 44 & 7,4 & 0,0 & 34,5 & 84,2 \\
\hline
\end{tabular}

cia y de infiltración cuya distribución varia a 10 largo del año. En cualquier caso destacar como el sector comprendido entre los transectos 1 y 2 siempre se comporta como área de surgencia a 10 largo del ciclo hidrológico y como el tramo comprendido entre los transectos 2 y 3 , se comporta como area de infiltración.

La variación del caudal a lo largo del año, tanto en la salida del humedal como en la charca lateral, está relacionada con el régimen de precipitaciones (Fig. 3). En ésta última el agua desaparece totalmente en septiembre. Los aportes de agua, via subsuperficial, desde el humedal al cauce del río son especialmente patentes aguas abajo del mismo, existiendo una relación significativa entre los caudales mensuales medidos en el transecto $7 \mathrm{y}$ los de salida de agua del humedal $(r=0.88, p=99.9 \%)$.

\section{Parametros morfológicos}

En la Tabla 1, se presentan los valores medios, máximo, minimo y coeficientes de variación para cada uno de los parámetros analizados. Como se
Tabla 2. Resultados del análisis de la varianza para los parámetros físico-químicos medidos en las estaciones del Rio Chicamo durante el periodo de estudio. $\left({ }^{*}\right)$ Datos transformados $(\log x+1) ; n$.s. $=$ no significativo. Results of variance analyses for physico-chemical parameters measured in the Chicamo stream during the study period. $(*)$ data transformed $(\log x+1) ; n$.s. $=$ no significant.

\begin{tabular}{lcc} 
& Kruskal-Wallis & ANOVA \\
\hline $\mathbf{p H}$ & $\mathbf{p}<0.001$ & \\
salinidad & & $\mathbf{p}<0.001$ \\
conductividad & & $\mathbf{p}<0.001$ \\
oxigeno disuelto & & $\mathbf{p}<0.001$ \\
sólidos suspensión* & & $\mathbf{p}<0.001$ \\
alcalinidad & $\mathbf{p}<0.001$ & $\mathbf{p}<0.001$ \\
nitratos & & $\mathbf{p}<0.001$ \\
nitritos* & n.s. \\
amonio* & & $\mathbf{p}<0.001$ \\
fosforo reactivo soluble* &
\end{tabular}

observa, se trata de un tramo de cauce muy ancho (30-50 m), como corresponde a un rio que sufre fuertes avenidas, donde la lamina de agua ocupa una pequeña proporción del mismo (entre el 15.8 $\%$ en octubre y el $6.2 \%$ en julio), de aguas muy 
someras y escaso caudal (valor medio de $3.3 \mathrm{~L} / \mathrm{s}$ ). En verano, el cauce queda estructurado en pequeiias pozas sin conexión aparente (superficial) y tanto la charca lateral como la salida del humedal quedan completamente secas.

\section{Parámetros físicos y quimicos}

La temperatura media del agua en el tramo de estudio fue de $18.6^{\circ} \mathrm{C}$, muy cercano al valor medio de la temperatura del aire para el periodo de estudio $\left(19^{\circ} \mathrm{C}\right)$ (Tabla 1$)$. El valor más elevado se detect6 en julio en la charca lateral $\left(30.5^{\circ} \mathrm{C}\right)$ (Fig. 4). Este parametro, para las estaciones del
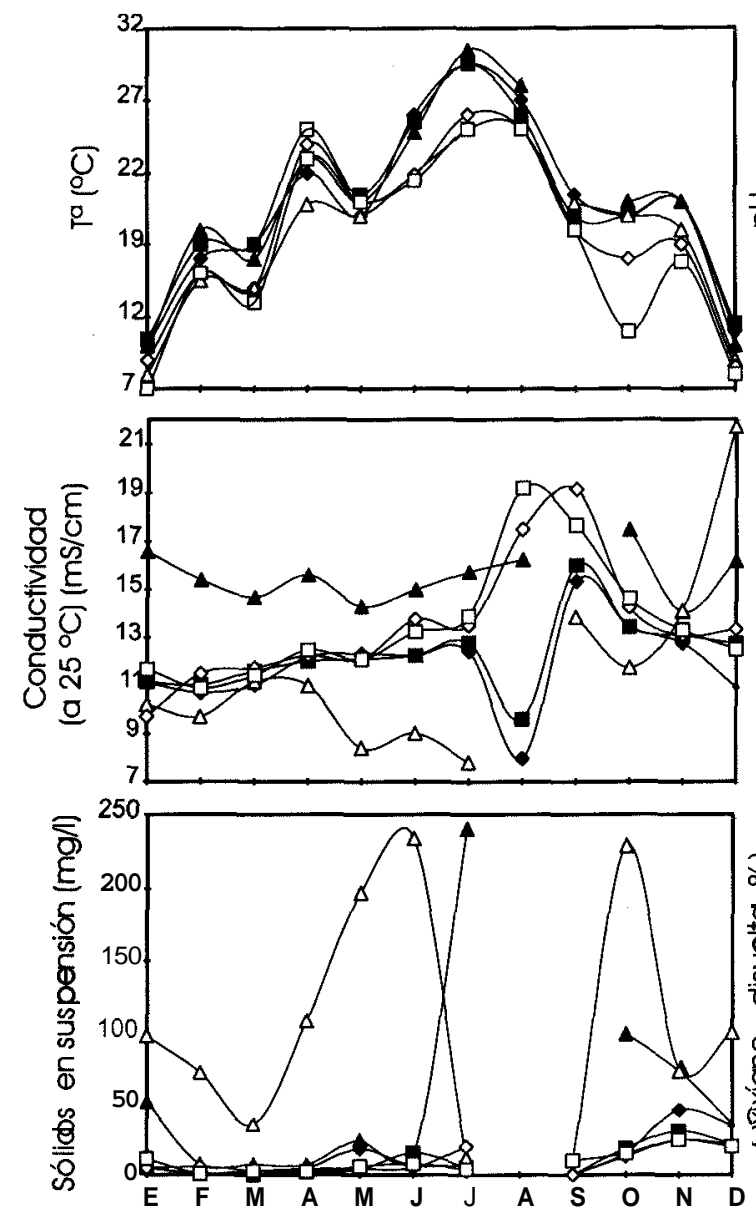

cauce principal, muestra una variación temporal significativa ( $<<0.001$; Tabla 2). Igual ocurre con el pH que, por el contrario no muestra variaciones espaciales importantes (Tabla 1).

En cuanto al oxigeno disuelto, las variaciones espaciales en el cauce principal no parecen muy importantes dado el valor del coeficiente de variación obtenido (Tabla 1). No ocurre igual con la variación temporal en la que se detectan diferencias significativas (Tabla 2 ). Durante todo el aiio se mantienen niveles altos de saturación de oxigeno (Fig. 4) en todas las estaciones de muestreo, excepto para la charca lateral y para la salida del humedal. La variación temporal de la satu-
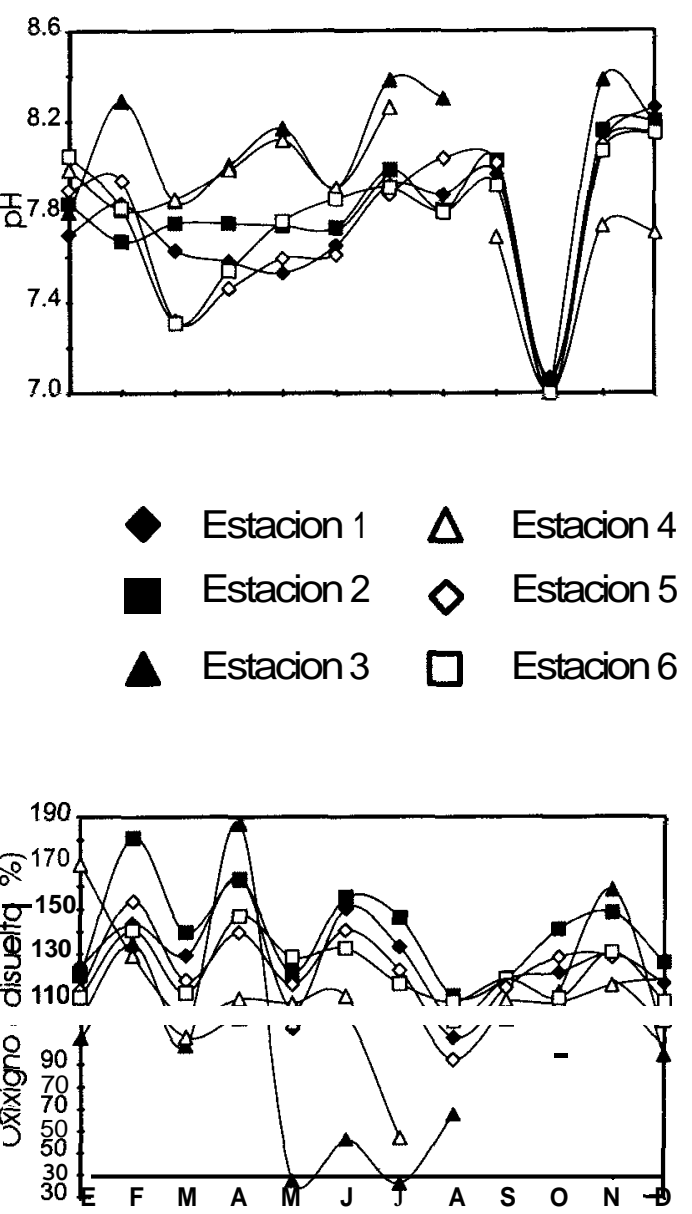

Figura 4. Valores mensuales de temperatura del agua ("C), conductividad a 25 "C (mS/cm), pH, saturación de oxigeno (\%) y sólidos en suspensión (mg/L), medidos en las estaciones de muestreo del Rio Chicamo. Monthly values of temperature of water $\left({ }^{\circ} \mathrm{C}\right.$ ), conductivity to $25^{\circ} \mathrm{C}$ $(\mathrm{mS} / \mathrm{cm}), \mathrm{pH}$, saturation of oxygen $(\%)$ and suspended solids $(\mathrm{mg} / \mathrm{L})$ measured in the sampling sites at Chicamo stream. 
ración de oxigeno esta relacionada con la temperatura del agua, con la que mantiene una relación negativa y altamente significativa $(\mathrm{r}=-0.48$, $\mathrm{p}=99.9 \%)$.

Los sólidos en suspensión muestran importantes variaciones tanto a nivel espacial como temporal (Tablas 1 y 2) (Fig. 4). La charca lateral y la salida del humedal presentan los valores más elevados $(56.22$ y $106.91 \mathrm{mg} / \mathrm{L}$, respectivamente).

La salinidad muestra variaciones temporales significativas $(\mathrm{p}<0.001$; Tabla 2$)$ debido a las os-
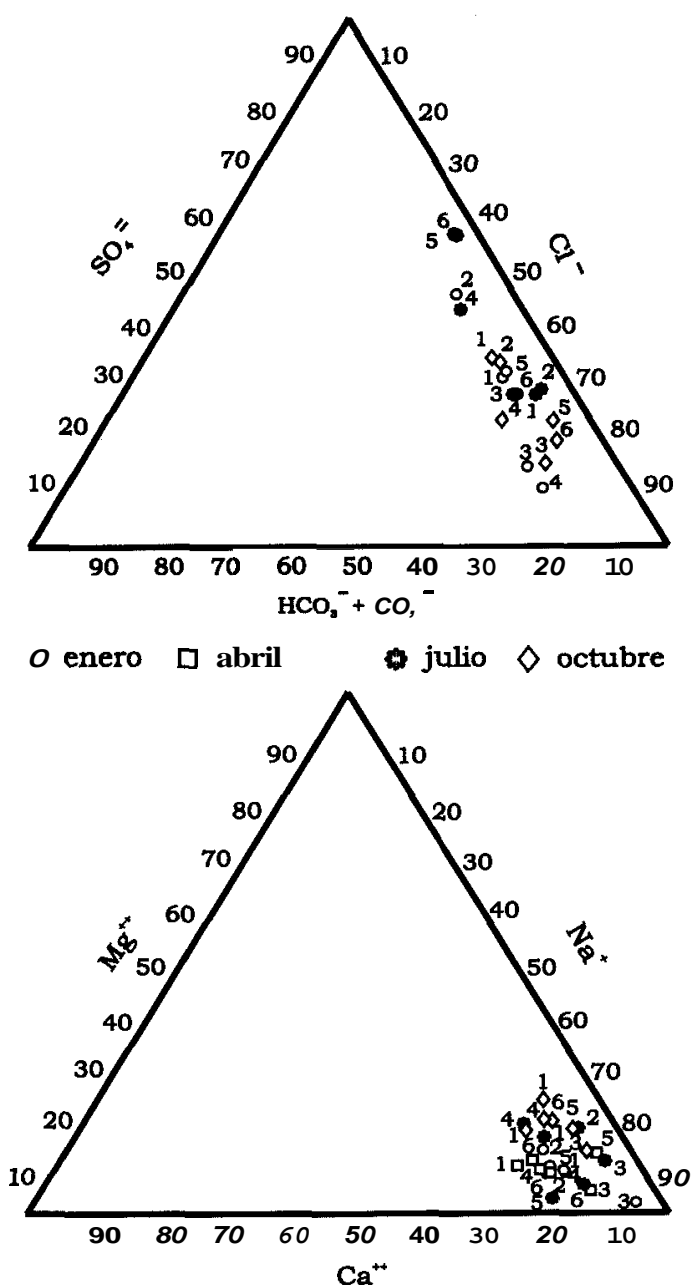

Figura 5. Diagramas triangulares de la composición aniónica y catiónica de las aguas superficiales del Rio Chicamo en el tramo de estudio. Relative anionic and cationic composition (triangular diagram) of the water at the sampling sites in the Chicamo stream. cilaciones de caudal, con el que guarda una relación negativa y significativa $(\mathrm{r}=-0.35 ; \mathrm{p}=99.9$ $\%)$. El valor medio para el cauce principal fue de $7.6 \mathrm{~g} / \mathrm{L}$ (Tabla 1). Teniendo en cuenta la clasificación propuesta por Montes \& Martino (1987) para las aguas salinas de cualquier origen, las del Rio Chicamo pueden calificarse como de aguas hiposalinas.

La conductividad $\left(\mathrm{a} 25^{\circ} \mathrm{C}\right)$ se comporta de forma similar a la salinidad, con un valor medio para las estaciones del cauce de $12.8 \mathrm{mS} / \mathrm{cm}$ (Fig. 4). Valores entre 12 y $36 \mathrm{mS} / \mathrm{cm}$, son habituales en las aguas de distintas ramblas, ríos y pequeiios humedales de la Cuenca del Rio Chicamo (VidalAbarca, 1985; Gómez, 1995), con lo que este tramo de cauce se situaría en el extremo inferior, mas dulce, de este rango.

El análisis de la composición aniónica y catiónica para las cuatro estaciones del aiio, refleja que las aguas del Rio Chicamo son bastante estables a 10 largo del tiempo (Tabla 1), con una composición cloruro-sódica dominante (Fig. 5). Sin embargo, en verano prevalecen los sulfatos frente a los cloruros, debido probablemente al efecto de la precipitación selectiva de sales (Carrasco et al., 1993).El valor medio de los cloruros para las estaciones del cauce es de $75.6 \mathrm{meq} / \mathrm{L}$, con un coeficiente de variación del $38.5 \%$.

La alcalinidad total proporciona valores medios para el cauce de $219.5 \mathrm{mg} / \mathrm{L}$ (Tabla 1). Se trata de aguas muy alcalinas (Margalef, 1977), en las que el sodio forma una parte importante del exceso de cationes, de hecho Cste es el dominante $(88.6 \mathrm{meq} / \mathrm{L})$, seguido por el magnesio $(21.3$ meq/L) , el calcio $(13.2 \mathrm{meq} / \mathrm{L})$ y finalmente el potasio $(0.3 \mathrm{meq} / \mathrm{L})$. Esta secuencia se encuentra también en las lagunas manchegas (Martino, 1988) y es la habitual en los sistemas acuáticos salinos del sureste ibérico (Gómez, 1995; Moreno et al., 1995).

En relación con los nutrientes, los valores medios para el nitrógeno en forma de nitratos, nitritos $\mathbf{y}$ amonio en el cauce principal fueron de 2.2, 0.03 y $0.3 \mathrm{mg} / \mathrm{L}$, respectivamente (Tabla 1 ). El N$\mathrm{NO}_{3}{ }^{-}$es, con diferencia la forma inorgánica de nitrógeno dominante en el Rio Chicamo. En general, la variación espacial de las distintas formas 
de nitrógeno parece ser tan importante e incluso más que la temporal, a excepción del $\mathrm{N}-\mathrm{NH}_{4}{ }^{+}$, donde no se detect6 una variación temporal significativa (Tabla 2) (Fig. 6).

Los valores más elevados de N-NO; siempre se detectan en la salida del humedal (valor máxi$\mathrm{mo}=23.8 \mathrm{mg} / \mathrm{L}$; valor minimo $=2.3 \mathrm{mg} / \mathrm{L}$ ), mientras que los de amonio en la charca lateral, sistemhticamente, durante todo el año (valor máximo $=29.2 \mathrm{mg} / \mathrm{L}$; valor mínimo $=2.3 \mathrm{mg} / \mathrm{L}$ ).
La variación temporal de $\mathrm{N}-\mathrm{NO}_{3} ; \mathrm{N}-\mathrm{NO} ; ; \quad \mathrm{N}$ $\mathrm{NH}_{4}{ }^{+}$parece relacionada con el ciclo hidrológico anual. En la Figura 7, se ha representado la proporción relativa (\%) de cada una de las fracciones de nitrógeno inorgánico disuelto (NID) para las seis estaciones de muestreo. Durante el verano, con el descenso del caudal, el aumento de la temperatura y la disminución del oxigeno disuelto, las concentraciones de N-NO; disminuyen, aumentando tanto las de N-NO; como las de N-
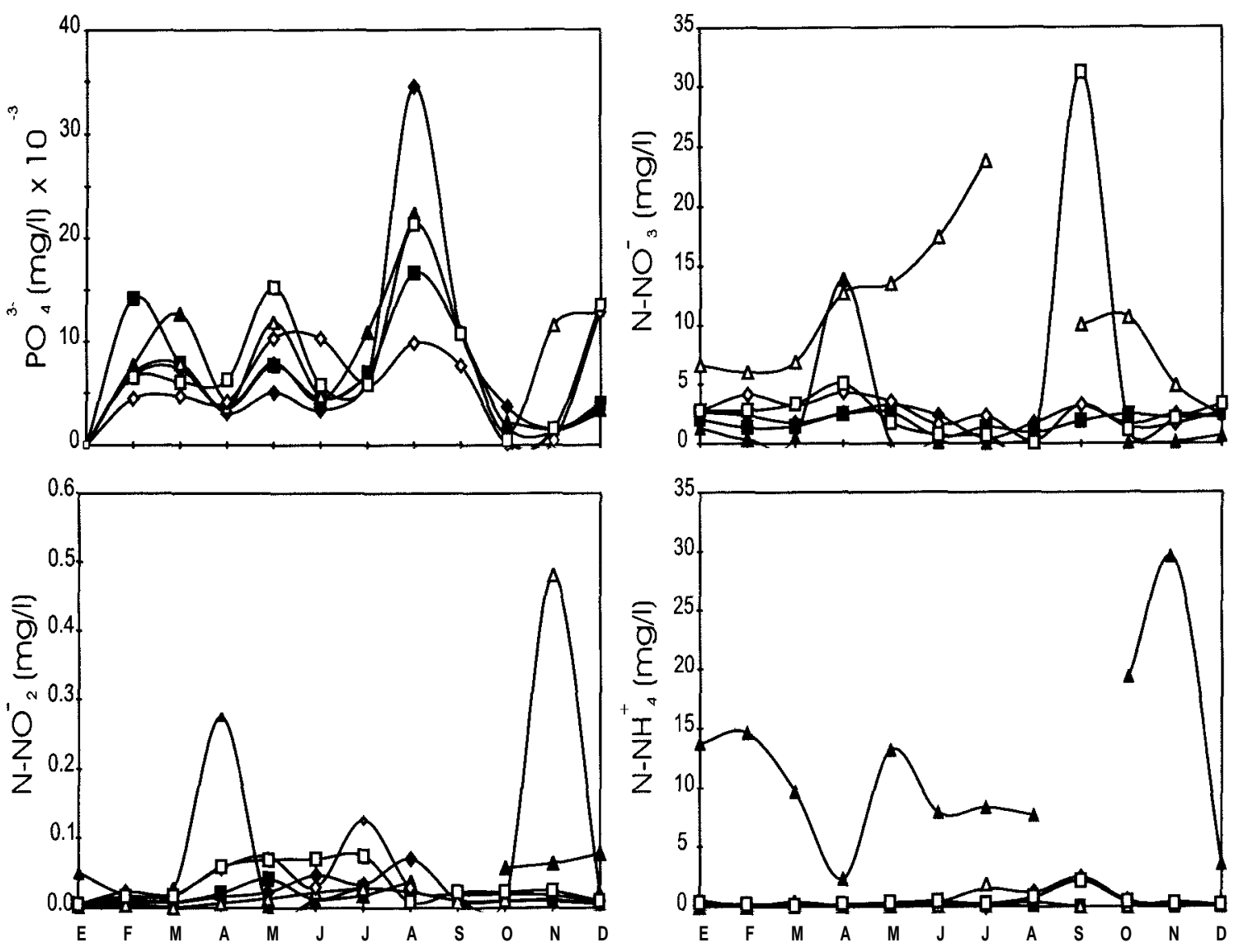

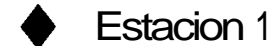

Stacion 1

$\triangle$ Estacion 4

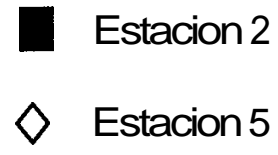

$\diamond$ Estacion 5

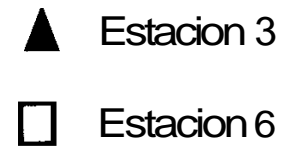

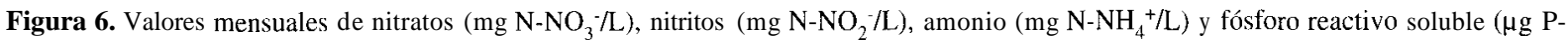

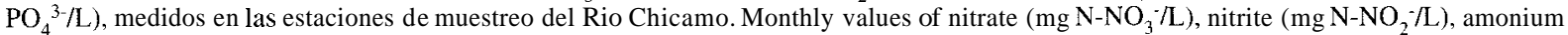
$\left(\mathrm{mg} \mathrm{N}-\mathrm{NH}_{4}{ }^{+} / \mathrm{L}\right)$ and $\mathrm{RSP}\left(\mu \mathrm{g} \mathrm{P}-\mathrm{PO}_{4}{ }^{3-} / \mathrm{L}\right)$, measured in the sampling sites at Chicamo stream. 
Tabla 3. Valores propios de las variables físico-químicas estudiadas, para los cuatro primeros ejes del PCA. Se han omitido los valores menores de 0,250. Eigen-values of the physico-chemical parameters for thefirstfour axes of PCA. Eigenvalues less than 0.250have been omitted.

EJEI EJE II EJEIII EJEIV

\begin{tabular}{lcccc}
\hline Anchura cauce & 0,472 & 0,252 & & \\
Caudal & 0,429 & $-0,287$ & & $-0,334$ \\
Temperatura agua & & 0,606 & $-0,385$ & \\
Saturación Oxigeno & 0,351 & & $-0,417$ & 0,453 \\
Conductividad & $-0,361$ & & & 0,255 \\
pH & & & 0,556 & 0,460 \\
P-PO4 & 0,261 & $-0,432$ & 0,524 & \\
N-NO3 & $-0,449$ & $-0,272$ & & 0,576 \\
N-NH4 & \% & & \\
\hline \%arianza acumulada & 29.3 & 46.4 & 61.1 & 71.7 \\
\hline
\end{tabular}

$\mathrm{NH}_{4}^{+}$. De hecho, existen relaciones significativas entre la concentración de N-NO; con la temperatura del agua $(r=0.48 ; p=99.9 \%)$ y con el oxigeno disuelto $(\mathrm{r}=-0.41 ; \mathrm{p}=99.9 \%)$. Este modelo, sin embargo, no se observa ni en la charca lateral ni en la salida del humedal.

En cuanto al PRS, el valor medio para el cauce fue de $7.4 \mu \mathrm{g} / \mathrm{L}$ (Tabla 1 ) y de 7.7 y $7.8 \mu \mathrm{g} / \mathrm{L}$ para la charca lateral y para la salida del humedal. Esta uniformidad que se observa en los valores medios para todas las estaciones de muestreo contrasta con la variación temporal significativa, al menos para las estaciones del cauce principal $(\mathrm{p}<0.001$; Tabla 2). El PRS (Fig. 6), experimenta un importante aumento al final del verano (agosto y septiembre) debido, probablemente a la disminucion de la lamina de agua y del flujo y al aumento de la descomposicion de la vegetación perifitica. Existen relaciones negativas y significativas entre el PRS y el caudal $(r=-0.27 ; p=95 \%)$ y el oxigeno disuelto $(\mathrm{r}=-0.43 ; \mathrm{p}=99.9 \%)$.

La relación $\mathrm{N}: \mathrm{P}$ es siempre muy superior a 16:1, de manera que no debe existir limitación por nitrógeno en el Rio Chicamo (Grimm \& Fisher, 1986).

\section{Análisis multivariante}

Los cuatro primeros ejes del PCA explican el $71.7 \%$ del total de la varianza. En la Tabla 3 se presentan los eigen-valores para dichos ejes asi como la contribución de cada uno de ellos al total de la varianza. El primer eje contrapone las variables relacionadas con la hidrologia (anchura de la lamina de agua y caudal) frente a la conductividad y la concentración de amonio. Podria interpretarse como un eje de mineralización. El segundo eje esta claramente relacionado con la temperatura del agua, indicando el efecto de la estacionalidad. El tercero, aunque está definido por varios parámetros, la saturación de oxigeno que se opone al $\mathrm{pH}$ y a la concentración de PRS, son los más importantes, quizás mostrando aspectos metabólicos del sistema. Por último, el cuarto eje esta definido sobretodo por la concentración de nitratos.
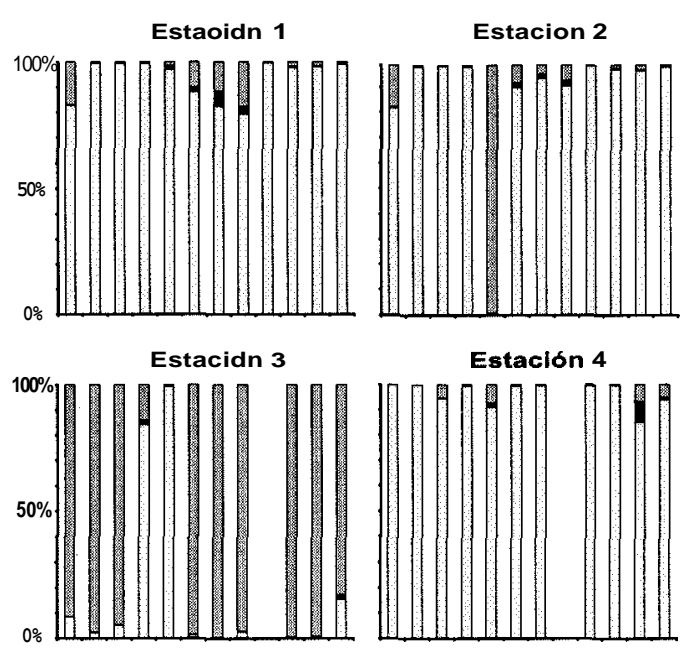

Estación 4
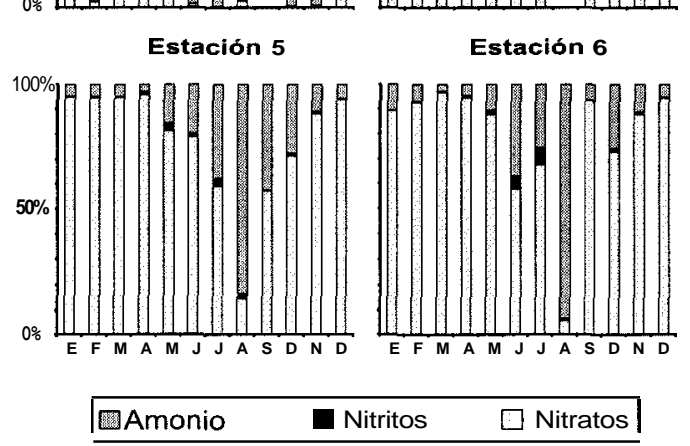

Figura 7. Proporción relativa (\%) de cada una de las fracciones de nitrógeno inorgánico disuelto (NID) medidos en las estaciones de muestreo del Rio Chicamo durante el periodo de estudio. Relative proportion (\%) of each one fraction of DIN measured in the sampling sites at Chicamo stream during the study period. 
En la figura 8 se presenta la posición de las muestras en el plano definido por los dos primeros ejes del PCA. La secuencia temporal que sigue cada una de las estaciones de muestreo permite observar una clara separación entre las estaciones 1 y 2 con respecto a la 5 y 6 . Parece existir un importante efecto temporal que segrega las estaciones del cauce situadas al final del tramo, donde el impacto de la "sequia" es mayor, frente a las situadas al inicio del mismo, donde se produce la "descarga" de agua subterránea.

Con el fin de poder establecer una secuencia temporal de fases o periodos dentro del ciclo hi- drologico anual para los dos extremos del cauce, se aplicó un análisis de clasificacion "cluster" sobre las coordenadas de los tres primeros ejes del PCA. En la figura 9, se muestra el dendrograma resultante, en el que es fácil diferenciar tres fases distintas. La primera se podria denominar "fase seca" que incluye a los meses desde mayo a septiembre para las estaciones 5 y 6 y desde junio a septiembre para las estaciones 1 y 2 . Parece existir un retardo en el proceso de sequia entre el principio y el final del tramo. Una segunda fase que podria denominarse "intermedia", incluye de forma indistinta a las cuatro estaciones de mues-

II

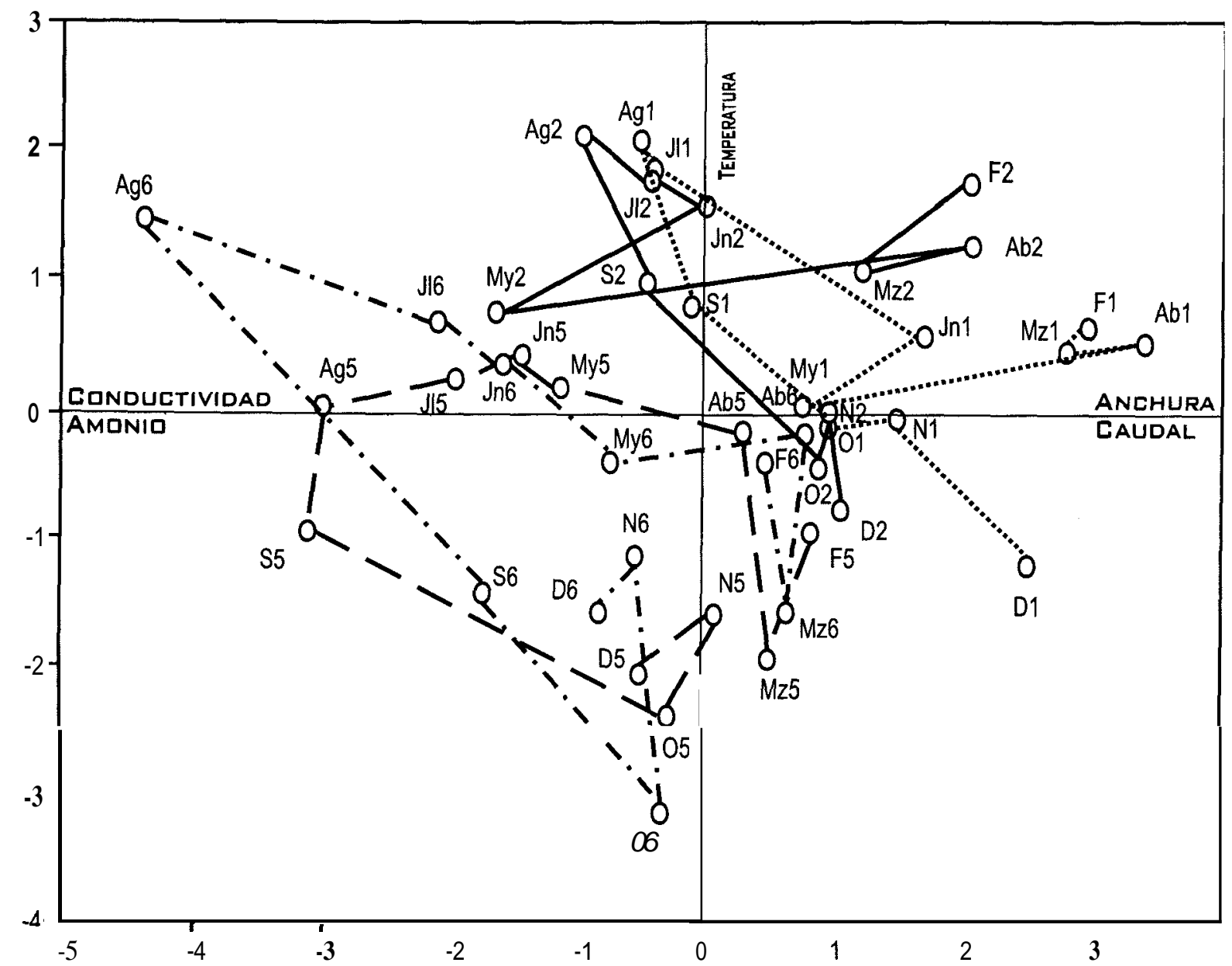

Figura 8. Proyección de las muestras y de los parámetros fisico-químicos medidos, en el plano definido por los dos primeros ejes del análisis de componentes principales (PCA). Samples and physico-chemical variables scores on thefirst two components of PCA. 


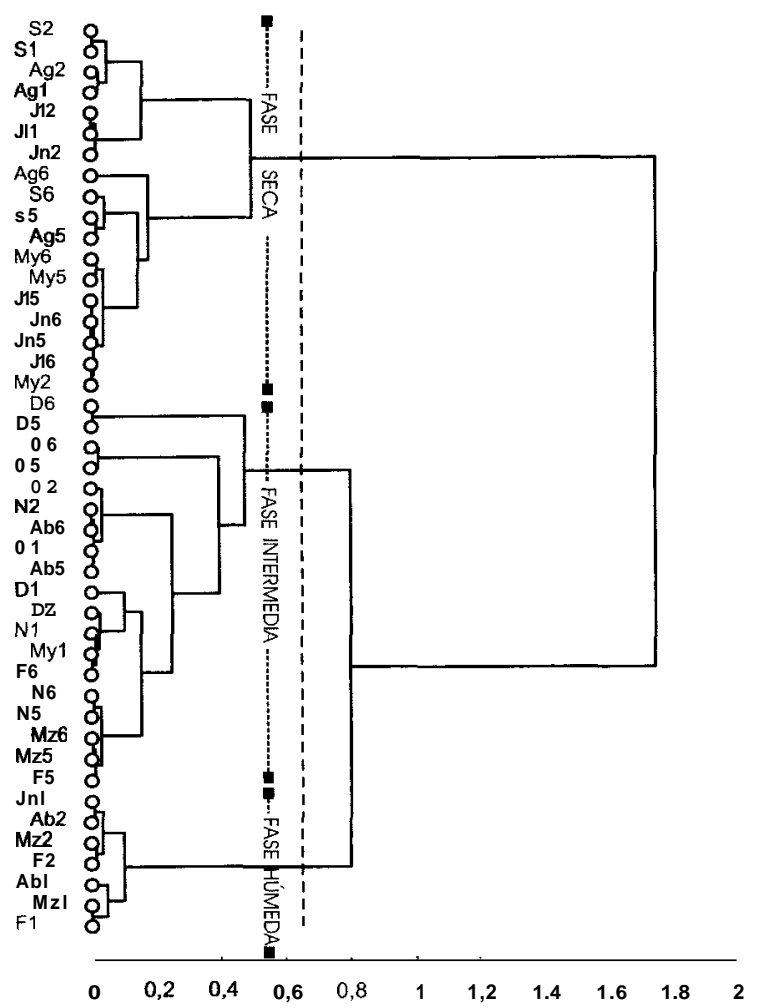

Figura 9. Dendrograma del análisis de clasificación realizado sobre las coordenadas de los tres primeros ejes del PCA. Dendrogram of the classification analysis with scores of the first three components of PCA.

treo, en los meses de primavera y de otoiio, es decir en los periodos del ciclo hidrológico donde se producen las lluvias. Finalmente una tercera fase que incluye unicamente a las estaciones de muestreo del inicio del tramo ( 1 y 2 ) en los meses del invierno y principio de la primavera donde se detectan los valores de caudal más altos, se podria denominar "fase humeda".

\section{DISCUSIÓN}

Cada vez es más evidente el cambio conceptual que ha sufrido la ecologia de rios que, en tan solo una dkcada ha pasado de estudiar las variaciones y procesos hidrológicos y ecológicos en el marco del "rio como un continuo" (Vannote et al., 1980), a reconocer la extraordinaria heterogenei- dad espacial y temporal de estos sistemas (Pringle et al., 1988). Esta situación se complica si cabe aún miis, cuando se trata de analizar los procesos que ocurren en rios de ambientes áridos y semihridos. Muchos estudios ponen de manifiesto la diversidad de hábitats que existen en estos sistemas (Jones et al., 1995; Stanley et al, 1997; Fisher et al., 1998b), y las interrelaciones existentes entre los subsistemas que componen el ecosistema fluvial (Fisher et al., 1998a). Asi por ejemplo, las interconexiones que se establecen entre el compartimento hiporreico y el superficial dependen, entre otras, de las propias características de la zona hiporreica (Brunke \& Gonser, 1997), la cual resulta imprescindible para explicar muchos procesos ecológicos.

La diniimica hidrológica superficial que se observa en el Rio Chicamo es compleja. Espacialmente la presencia de rezumes laterales y la gran cantidad de pequeiias fracturas que se observan en el sustrato de margas del lecho, podría explicar las zonas de surgencias y de infiltración (Fig. 2). Los materiales margosos siempre se han interpretado como "impermeables", pero la gran cantidad de pequeiias fracturas que se observan en el cauce del Rio Chicamo podrian explicar este comportamiento hidrológico.

A lo largo del ciclo hidrológico, apenas se observa relación entre la curva de variación de las precipitaciones (Fig. 3) y el modelo hidrológico observado (Fig. 2). Gordon et al. (1992) explican cómo los rios intermitentes que reciben agua sólo en determinadas epocas del aiio, pueden actuar como rios de descarga o de recarga dependiendo de la estación del aiio. En la cuenca del Chicamo muchas lluvias locales, a veces de gran intensidad, que no son recogidas en los observatorios de la red termopluviomktrica podrian explicar, en parte, estos comportamientos hidrológicos.

Una de las singularidades de los rios de las regiones áridas es su tendencia a ser salinos. La salinidad de las aguas del Rio Chicamo tiene su origen en las margas miocénicas que cubren buena parte de la Cuenca del Rio Chicamo (Aguilar \& Gallardo, 1989) que aportan sulfatos a las aguas de escorrentía. Sin embargo, el carácter 
clorurado-sódico dominante de las aguas del Rio Chicamo debe tener su origen en otros procesos diferentes.

La variación de los valores de salinidad en el tramo de cauce estudiado podria estar relacionada, en primer lugar, con la hidrologia del río. De hecho existe una relación opuesta y significativa entre la salinidad y el caudal $(\mathrm{r}=-0.35, \mathrm{p}=99.9$ $\%)$. Además, la entrada puntual de agua subsuperficial, más dulce o bien mas salada, también podria explicar estas variaciones a nivel espacial, como 10 demuestra Gomez (1995), para pequeños humedales de la Cuenca del Chicamo e, igualmente, los procesos de evaporación-cristalización-redisuolución, podrían ser responsables de las variaciones temporales observadas (Kilham, 1990). En ambientes áridos, sometidos a una intensa evaporación, como es el caso del Rio Chicamo, los cloruros, de elevada solubilidad, aumentan su concentracion al hacerlo la evaporación, pero no llegan a producirse fenómenos de precipitación como ocurre con los carbonatos o los sulfatos con productos de solubilidad menores.

El proceso secuencial de precipitación salina es complejo (Martino, 1988), asi los minerales de calcio son los primeros en precipitar (calcita: $\mathrm{CaCO}_{3}$ y dolomita: $\left.\mathrm{CaMg}\left(\mathrm{CO}_{3}\right)_{2}\right)$. Al incrementarse la concentración salina al hacerlo la evaporación, el yeso $\left(\mathrm{CaSO}_{4} \cdot 2 \mathrm{H}_{2} \mathrm{O}\right)$ y la anhidrita
$\left(\mathrm{CaSO}_{4}\right)$, de solubilidad relativamente baja, precipitan tambikn (Borchert, 1965), de manera que el sulfato restante se asocia al magnesio y al sodio para formar epsomita $\left(\mathrm{MgSO}_{4} .7 \mathrm{H}_{2} \mathrm{O}\right) \mathrm{y}$ mirabilita $\left(\mathrm{Na}_{2} \mathrm{SO}_{4} .10 \mathrm{H}_{2} \mathrm{O}\right)$, que no es lavada por las Iluvias, y se acumula en el suelo. AI deshidratarse forma un polvo blanquecino (Pizarro, 1985) visible durante primavera y sobre todo en verano en el Rio Chicamo.

Otra singularidad del Rio Chicamo se refiere a las altas concentraciones de N-NO;; detectadas (más de $2 \mathrm{mg} / \mathrm{L}$, como media). Aunque generalmente se atribuyen a influencias antrópicas agrícolas y ganaderas (Peterjohn \& Correll, 1984; Lowrance et al., 1985; Hill, 1988), en areas margosas y ricas en evaporitas, como ocurre en esta cuenca, es esencial el papel de los nitratos sedimentarios que caracterizan a los depositos evaporíticos (Gonzalez-Bernaldez, 1989). La importancia de la nitrofília en Breas margosas y ricas en evaporitas, observada por muchos botánicos, explicaría la doble especialización (halofílica y nitrofílica) de algunas especies de helofitos típicos de estos sistemas (Gómez, 1995).

La dominancia de una u otra forma de nitrógeno inorgánico (N-NO;; N-NO; $\quad \mathrm{N}-\mathrm{NH}_{4}^{+}$) en las aguas del Rio Chicamo, dependera del estado de oxidación-reducción que prevalezca en cada momento, pero no solo en el agua superficial sino también en los sedimentos (DAHM, et al., 1998).

Tabla 4. Valores medios, máximos, minimos y desviación estandar de los principales parámetros físico-químicos que caracterizan las distintas fases hidrológicas definidas para el Rio Chicamo. Mean, muxinzum, minimum values and standard deviation of the main physico-chemical characteristics of each phases of flow in the Chicamo streum.

\begin{tabular}{|c|c|c|c|c|c|c|c|c|c|c|c|c|c|c|c|c|}
\hline & \multirow[b]{2}{*}{$\begin{array}{l}\mathrm{N}=7 \\
\text { media }\end{array}$} & \multirow[b]{2}{*}{$\operatorname{Max}$} & \multirow[b]{2}{*}{ Min } & \multirow[b]{2}{*}{ SD } & \multicolumn{4}{|c|}{ (abajo) } & \multicolumn{3}{|c|}{ FASE HUMEDA } & \multicolumn{5}{|c|}{ FASE INTERMEDIA } \\
\hline & & & & & $\begin{array}{l}\mathrm{N}=11 \\
\text { media }\end{array}$ & Max & Min & SD & $\begin{array}{l}\mathrm{N}=7 \\
\text { media }\end{array}$ & $\operatorname{Max}$ & Min & SD & $\begin{array}{l}\mathrm{N}=19 \\
\text { media }\end{array}$ & Max & Min & SD \\
\hline Anchura lamina agua (m) & 8,0 & 9.3 & 6.6 & 0,9 & 3,5 & 8.9 & 2,3 & 1,9 & 16.3 & 22,5 & 10,0 & 5,7 & 8.5 & 20,4 & 3.1 & 6,7 \\
\hline Caudal $(1 / s)$ & 0,8 & 1.4 & 0,0 & 0,5 & 1,1 & 4.8 & 0,0 & 1,3 & 6.3 & 13,1 & 1,2 & 4,6 & 4,2 & 15,9 & 1.0 & 3,4 \\
\hline Temperaturd Agua $\left({ }^{\circ} \mathrm{C}\right)$ & 25,3 & 29,5 & 19,0 & 4,1 & 21,9 & 26,0 & 18.0 & 2,9 & 20.0 & 26,0 & 16,0 & 4,2 & 15,8 & 24,0 & 8,0 & 4,6 \\
\hline Satur. Oxig (\%) & 126,5 & 155,1 & 101,7 & 19.2 & 119,5 & 140,7 & 92,0 & 12,7 & 153,0 & 181,0 & 129,3 & 17.5 & 127,2 & 153,5 & 105,7 & 14,8 \\
\hline Conduct. $\left(25^{\circ} \mathrm{C}\right)(\mathrm{mS} / \mathrm{cm})$ & 12,3 & 16,0 & 8,0 & $2 Y$ & 14,9 & 19.2 & 12.1 & 2,9 & 11,5 & 12,3 & 10,7 & 0,6 & 12,6 & 14,6 & 10,9 & 1,0 \\
\hline $\mathrm{pH}$ & 7.9 & 8,0 & 7,7 & 0,1 & 7,8 & 8,0 & 7.6 & 0,1 & 7,7 & 7,8 & 7,6 & 0,1 & 7,7 & 8.3 & 7,0 & 0.5 \\
\hline PRS ( $\mu \mathrm{g}$ P-PO4/L) & 12.9 & 34,5 & 4.4 & 10,3 & 9,4 & 21.3 & 3.3 & 5,1 & 6,5 & 14.1 & 3.1 & 3,9 & 4,4 & 13.5 & 0,0 & 3,8 \\
\hline Nitratos (mgN-NO3L) & 1,4 & 3,2 & 0,4 & 0.9 & 4,0 & 31,2 & 0.0 & 9,1 & 2,0 & 25 & 1.3 & 0,5 & 2,7 & 5,1 & 1,2 & 1.0 \\
\hline Amonio (mg N-NH4/L) & 0,1 & 0,4 & 0,0 & 0.1 & 2,1 & 13.2 & 0,3 & 3,8 & 0,0 & 0,3 & 0,0 & 0,1 & 0,2 & 0,5 & 0.0 & 0.1 \\
\hline
\end{tabular}


Los valores más elevados de N-NO; siempre se detectan en la salida del humedal, debido a la existencia de una fuente al final del mismo (Gómez, 1995). Por el contrario, en la charca lateral siempre se detectan los valores más altos de amonio, debido probablemente a la presión del ganado ovino que soporta.

En el cauce principal, la compleja dinámica hidrológica del Rio Chicamo, en relación al efecto de "fuente" o "sumidero" de agua superficial, podria explicar las variaciones en las concentraciones de nitratos y sobre todo amonio al menos, entre los transectos 1 y 3 . Así, en la primera zona de surgencia se midieron valores medios de $\mathrm{N}$ $\mathrm{NO} ;$ y N-NH${ }_{4}^{+}$más elevados $(0.02$ y $0.12 \mathrm{mg} / \mathrm{L}$, respectivamente) que los detectados en el área de infiltración $(0.016$ y $0.03 \mathrm{mg} / \mathrm{L}$, respectivamente). Valet, et al. (1994) encuentran valores más altos de nitratos en zonas de cauce de "upwelling" (áreas de surgencia) en un río del desierto de Arizona (Sycamore Creek) limitado por el nitrógeno, al igual que Maltchick (1994) en un arroyo temporal de Córdoba (Arroyo de la Montesina). Las descargas de agua subterranea, o que han discurrido por las capas aluviales hiporreicas de los cauces, son especialmente ricas en N-NO,. Sin embargo, no siempre ocurre esto. En los estudios antes mencionados, el sustrato del cauce estaba constituido por materiales de textura gruesa (gravas y arenas) en los que los espacios intersticiales permiten la oxigenación, favoreciendo, entre otros, la nitrificación $\left(\mathrm{N}_{-} \mathrm{NH}_{4}{ }^{+} \rightarrow\right.$ $\mathrm{N}-\mathrm{NO}_{3}{ }^{-}$) (Holmes et al., 1994), sin embargo, en sustrato arcillo-limosos, como es el del Rio Chicamo, los espacios intersticiales son muy escasos dada la facilidad de compactación de estos materiales. En consecuencia hay un déficit de oxigeno y los procesos hidroquimicos se realizan en condiciones anaeróbicas, al igual que, por ejemplo, la reduccidn de N-NO; a N-NH${ }_{4}^{+}$(Triska et al., 1993, Pinay et al.,1994; Brunke \& Gonser, 1997).

Finalmente, la interpretación de los análisis de ordenación y clasificación realizados sobre la matriz de datos físico-químicos permite identificar, al menos, tres fases hidrológicas (Fig. 9) caracterizadas por la variación de algunas variables físico-químicas. En la Tabla 4 se ha calculado, para cada una de ellas los valores medios, maximo, minimo y la desviación estandar de los parámetros fisicos y quimicos mas significativos. Durante la fase seca se produce un efecto de "contracción" del sistema (Stanley et al., 1997), de manera que en ella se miden los valores minimos de anchura de la lamina de agua, de caudales $(0.8$ y I. $1 \mathrm{~L} / \mathrm{s})$ y de saturación de oxigeno (119.5\%), al contrario que para la temperatura, la conductividad y las concentraciones de PRS y de $\mathrm{N}-\mathrm{NH}_{4}{ }^{+}$, para los que se detectan los valores mas altos $\left(25.3^{\circ} \mathrm{C} ; 14.9 \mathrm{mS} / \mathrm{cm} ; 12.9 \mu \mathrm{g} / \mathrm{L} ; 2.08 \mathrm{mg} / \mathrm{L}\right.$, respectivamente) puesto que esta fase coincide con la época de verano, en la que los procesos de descomposición de la materia orgánica son muy activos, junto a un efecto de concentración por la disminución del caudal.

Durante la fase humeda se detecta la mayor cobertura de la lamina de agua $(16.3 \mathrm{~m})$, los valores máximos de caudal $(6.3 \mathrm{~L} / \mathrm{s})$ y de saturación de oxigeno $(153 \%)$ y los minimos de $\mathrm{N}-\mathrm{NH}_{4}{ }^{+}$ $(0.04 \mathrm{mg} / \mathrm{L})$. Se trata de una fase "expansiva", según Stanley et al. (1997) y progresiva, que implica un proceso de uniformización y homegeneización de las condiciones ambientales (Gómez et al., 1995). Esta fase solo se detecta en las estaciones del inicio del tramo ( 1 y 2 ), probablemente porque desde este punto se restituyen las condiciones hidricas de todo el tramo tras las lluvias. Por ultimo, la fase intermedia que incluye las épocas del ciclo hidrológico de mayor inestabilidad (otoiio y primavera), se caracteriza por los valores también intermedios para casi todos los parámetros analizados.

Las tres fases definidas corresponden al año de muestreo (1994) pero la variabilidad del régimen de precipitaciones en el área de estudio, permite suponer que tanto la duración de cada una de las fases como la intensidad de los parametros analizados, en términos de concentración, variaran considerablemente a una escala interanual.

Es necesario seguir trabajando sobre los aspectos relacionados con los procesos ecológicos que caracterizan los nos más aridos. Aun cuando las previsiones de cambio climático hacia la acentuación de las condiciones ambientales de aridez, no sean cstrictamente ciertas, si 10 es el que estamos 
asistiendo a la extracción exhaustiva de agua tanto superficial como subterranea en todo tipo de ecosistema acuatico. En consecuencia, la intermitencia y la temporalidad de nuestros ríos sera previsiblemente mayor cada dia y también la necesidad de conocer su funcionamiento.

\section{AGRADECIMIENTOS}

El grupo de investigadores que recogia las muestras era más amplio que el numero de autores de este trabajo: agradecemos la colaboracidn de Josefa Velasco, Andrés Millán; Cristina Guerrero, Bernardo Martinez, y Alberto Perán. Igualmente agradecemos las indicaciones de dos correctores andnimos que nos animaron a rehacer e incorporar más informacidn a este trabajo. Los datos termopluviométricos han sido proporcionados por el Instituto Meteorologico del Sureste (Ministerio de Medio Ambiente). Los resultados de este estudio han sido financiados por el Proyecto PB961113 de la Comisión Interministerial de Ciencia y Tecnologia.

\section{BIBLIOGRAFÍA}

AGUILAR, J. y J. GALLARDO. 1989. Tipologias y variación de tipos de suelos en zonas áridas. En: Zonas aridas en España. Real Academia de Ciencias Exáctas, Fisicas y Naturales (ed.): 287-305. Madrid.

A.P.H.A. 1985. Standard methodsfor the examination of water and waste water. 16th ed. Amer. Public Health Assoc., Inc. Washington.

BORCHERT, H. 1965. Principles of oceanic salt deposition and metamorphism. In: Chemical Oceanography 11.J.P. Riley \& G. Skirrow (eds.): 205-276. Academic Press. N.Y.

BOULTON, A.J. \& P.J. SUTER. 1986. Ecology of temporary stream. An australian perspective. In: Limnology in Australia. Deckker, P. \& W. D. Williams (eds.): 313-327. CSIRO/Dr. Junk. Melbourne.

BOULTON, A.J. \& P.S. LAKE. 1990. The ecology of two intermittent streams in Victoria, Australia.I. Multivariate analyses of physicochemical features. Freshwat. Biol.,24: 123-141.
BRUNKE, M. \& T. GONSER. 1997. The ecological significance of exchange processes between rivers and groundwater. Freshwat. Biol.,37: 1-33.

CARRASCO, F., I. GAVILÁN \& P. RODRÍGUEZ. 1993. Evolucidn quimica en un año humedo del agua de la Laguna de Fuente de Piedra (Prov. De Málaga). Actas VI Congr. Esp. Limnol.: 69-74.

COMIN, EA. \& W.D. WILLIAMS. 1994. Parched continents: Our common future?. In: Limnology now: A paradigm of Planetary Problems. Margalef, R. (ed.): 473-527. Elsevier Sc. B.V.

DAHM, C.N., N.B. GRIMM, P. MARMONIER, H.M. VALETT \& P. VERVIER. 1998. Nutrient dynamics at the interface between surface waters and groundwaters. Freshwat. Biol. 40: 427-451.

DODDS, W.K., J.M. BLAIR, G.N. HENEBRY, J.K. KOELLIKER, R. RAMUNDO \& C.M. TATE. 1996. Nitrogen transport from tallgrass pairie watersheds. J. Environ. Qual.,25: 973-981.

FAHMY, T. 1999. XLSTAT. Version 4.0.

FISHER, S.G. \& W.L. MINKLEY. 1978. Chemical characteristics of a desert stream in flash flood. $J$. Arid Environm., I: 25-33.

FISHER, S.G., L.J. GRAY, N.B. GRIMM \& D.E. BUSCH. 1982. Temporal sucession in a desert stream ecosystem following flash flooding. Ecol. Mong., 52: 93-110.

FISHER, S.G., N.B. GRIMM, E. MARTI, R.M. HOLMES \& J.B. JONES, Jr. 1998a. Material spiraling in stream corridors: A telescoping ecosystem model. Ecosystems, 1: 19-34.

FISHER, S.G., N.B. GRIMM, E. MARTI \& R. GOMEZ 1998b. Hierarchy spatial configuration, and nutrient cycling in a desert stream. Austral. J. Ecol., 23: 41-52.

GOLTERMAN, H.L., R.S. CLYMO \& M.A.M. OHNSTAD. 1978. Methods for physical and chemical analysis of freshwaters. IPB Handbook $n^{\circ} 8$. Blackwell Sc. Publ. Oxford.

GOMEZ, R. 1995. Funcidn de los humedales en la dinamica de nutrientes ( $N$ y $P$ ) de una cuenca de caracteristicas aridas: Experiencias en el sureste ibérico. Tesis Doctoral. Universidad de Murcia. 300 pp.

GOMEZ, R., R. VIDAL-ABARCA, M.L SUAREZ \& S.G. FISHER. 1995. The spatial and temporal scale problem in arid zones wetlands management. In: Buses ecológicas para la restauracidn de humedales en la cuenca mediterránea. C. Montes, G. Oliver, F. Molina \& J. Cobos (eds.): 95-105. Junta de Andalucia. Sevilla. 
GONZALEZ-BERNALDEZ, F. 1989. Ecosistemas áridos y endorreicos espaiioles. En: Zonas áridas en España. Real Academia de Ciencias Exáctas, Físicas y Naturales (ed.): 223-238. Madrid.

GORDON, N.B., T.A. McMAHON \& B.L. FINLAYSON. 1992. Stream Hydrology. An introductionfor ecologists. Wiley. Chichester.

GRIMM, N.B. \& S.G. FISHER. 1986. Nitrogen limitation in a Sonoran desert stream. J. N. Am. Benthol. Soc., 5: 2-15.

HILL, B.H. 1988. Factors influencing nitrate depletion in a rural stream. Hydrobiologia, 160: 111-122.

HOLMES, R.M., S.G. FISHER \& N.B. GRIMM. 1994. Parafluvial nitrogen dynamics in a desert stream ecosystem. J. N. Am. Benthol. Soc., 13: 468478.

JONES, J.B., S.G. FISHER \& N.B. GRIMM. 1995. Vertical hydrologic exchange and ecosystem metabolism in a Sonoran desert stream. Ecology, 76: 942-952.

KILHAM, P. 1990. Mechanisms controlling the chemical composition of lakes and rivers: Data from Africa. Limnol. Oceanogr., 35: 80-83.

LOWRANCE, R., R. LEONARD \& L. ASMUSSEN. 1985. Nutrient budgets for agricultural watersheds in the southeastern coastal plain. Ecology, 66(I): 287-296

MACKERETH, F.J.H., J. HERON \& J.F. TALLING. 1978. Water Analyses. Some methods for limnologists. Freshwat. Biol. Asoc. Sc. Publ., n 36 . Cumbria.

MALTCHIK, L. 1994. Dinámica de nutrientes en un arroyo temporal mediterraneo ( $S W$, Cdrdoba). Tesis Doctoral. Universidad Autdnoma de Madrid. $210 \mathrm{pp}$.

MALTCHIK, L., \& S. MOLLA. 1994. Nutrient dynamics in hyporheic zone in a mediterranean stream in Sierra Morena (SW Spain). Verh. Internut. Verein Limnol., 25: 2434-2437.

MALTCHIK, L., S. MOLLA, C. CASADO \& C. MONTES. 1994. Measurement of nutrient spiralling in a mediterranean stream: Comparison of two extremes hydrologic phases. Arch. Hydrobiol., 130: 215-227.

MALTCHIK, L., S. MOLLA \& C. CASADO. 1998. Spatio-temporal heterogeneity of nutrients in a mediterranean stream. (Montesina stream, SW, Spain). Verh. Internat. Verein Limnol.,26: 10621065.

MARTI, E. \& F. SABATER. 1996. High variability in temporal and spatial nutrient retention in mediterranean streams. Ecology, 77: 854-869.
MARTINEZ, B., J. VELASCO, M.L SUAREZ \& M.R. VIDAL-ABARCA. 1998. Benthic organic matter dynamics in an intermittent stream in southeast Spain. Arch. Hydrobiol., 141: 303-320.

MARTINO, P. 1988. Limnologia de las lagunas salinas espaiiolas. Tesis Doctoral. Universidad Autónoma de Madrid. 264 pp.

MARGALEF, R. 1977. Ecologia. Omega.

MATTHEWS, W.J. 1988. North American praire streams as systems for ecological study? J. N. Am. Benthol. Soc., 7: 387-409.

MOLLA, S., L.MALTCHIK \& C. CASADO. 1994. Primeros datos sobre el metabolismo de un arroyo temporal mediterráneo de Sierra Morena (Córdoba). Limnetica, 10: 59-67.

MONTES,C. \& P. MARTINO. 1987. Las lagunas salinas españolas. En: Bases científicas para la proteccidn de los humedales en Espaiia. Real Academia de Ciencias Exáctas, Físicas y Naturales (ed.): 95145. Madrid.

MORENO, J.L., M.L. SUAREZ \& M.R. VIDALABARCA. 1995. Hidroquimica de las ramblas litorales de la Región de Murcia: Variaciones espaciotemporales. Limnetica, 11 (1): 1-13.

ORTEGA, M., M.R. VIDAL-ABARCA, M.L. SUAREZ, J.L. GONZALEZ-BESERAN \& L.. RAMIREZ-DIAZ. 1988. Caracteristicas fisico-quimicas de las aguas superficiales de la Rambla del Moro despues de una riada (Cuenca del Rio Segura, SE de Espaiia). Limnetica, 4: 19-26.

PETERJOHN, W.T. \& D.L. CORRELL. 1984. Nutrient dynamics in an agricultural watershed: Observations on the role of a riparian forest. Ecology, 65: 1466-1475.

PINAY,G., N.E. HAYCOCK, C. RUFFINONI \& R.H. HOLMES. 1994. The role of denitrification in nitrogen removal in river corridors. In: Global Wetlands: Old and New World. W. J. Mitsch (ed) : 107-116. Elsevier. Amsterdam.

PIZARRO, F. 1985. Drenaje agricola y recuperación de suelos salino. 2nd.Ed. Ed. Agricola Espaiiola. S.A. Madrid.

PRINGLE, C.M., R.J. NAIMAN, G. BRETSCHKO, J.R. KARR, M.W. OSWOOD, R.J. WEBSTER, R.L WELCOMM \& M.J. WINTERBOURN. 1988. Patch dynamics in lotic systems: The stream as a mosaic. J. N. Am. Benthol. Soc., 7: 503-524.

SABATER, F. \& J. ARMENGOL. 1986. Chemical characterization of the Ter River. Limnetica, 2: 75-84.

SABATER, F., J. ARMENGOL \& S. SABATER. 1991. Physical and chemical disturbance associated 
with spatial and temporal variation in a mediterranean river. J. N. Am. Benthol. Soc., 10: 2-13.

SABATER, S., H. GUASCH, E. MARTI, J. ARMENGOL, M. VILA \& F. SABATER. 1992. The Ter, a mediterranean river system in Spain. In: Limnology in Spain. C. Montes, C. Duarte \& J. Garcia-Aviles (eds.): 141-150. Asociacidn Española de Limnologia. Granada.

STANLEY, E.H. \& S.G. FISHER. 1992. Intermittency, disturbance, and stability in stream ecosystem. In: Aquatic ecosystems in semi-arid regions: implicationsfor resource management. R.D. Robarts \& B.L. Bothwell (eds.): 234-249. N.H.R.I. Symposium Sr.7. Environment Canadá. Saskatoon Canada.

STANLEY,E.H. \& A.J. BOULTON. 1995. Hyporheic processes during flooding and drying in a Sonoran desert stream. I. Hydrologic and chemical dynamics. Arch. Hydrobiol., 134: 1-26.

STANLEY, E.H., S.G. FISHER \& N.B. GRIMM. 1997. Ecosystem expansion and contraction in stream. BioScience, 47: 427-435.

TRISKA, F.J., J.H. DUFF \& R.J. AVANZINO. 1993. The role of water exchange between a stream channel and its hyporheic zone in nitrogen cycling at the terrestrial-aquatic interface. Hydrobiologia, 251 : 167-184.

VALETT. H.M., S.G. FISHER \& E.H. STANLEY. 1990. Physical and chemical characteristics of hyporheic zone of a Sonoran Desert stream. J. N. Am. Benthol. Soc., 9:201-215.

VALETT. H.M., S.G. FISHER, N.B. GRIMM \& P. CAMILL. 1994. Vertical hydrologic exchange and ecological stability of a Sonoran Desert stream. Ecology, 75: 548-560.

VANNOTE, R.L., G.W. MINSHALL, K.W. CUMMINS, J.R. SEDELL \& C.E. CUSHING. 1980. The river continuum concept. Can. J. Fish. Aquat. Sci., 37: 130-137.

VIDAL-ABARCA, M.R. 1985. Las aguas superficiales de la Cuenca del Rio Segura (SE de España). Caracterizacidn físico-química en relacidn al medio físico y humano. Tesis Doctoral. Universidad de Murcia. 789 pp.

VIDAL-ABARCA, M.R., C. MONTES, M.L. SUAREZ \& L. RAMIREZ-DIAZ. 1987. El clima de la Cuenca del Rio Segura (SE de Espafia). Factores que lo controlan. Anales de Biología, 12: 11-28.

WETZEL, R.G.\& G.E. LIKENS. 1991. Limnological analyses. Springer Verlag. Berlin.

WINKLER, L.W. 1888. Die bestimmung des im wasser gelodten saverstoffs. Ver. Dtsch. Chem. Ges. 21: 2843-2954.

ZALE, A.V., D.M. LESLIE, W.L. FISHER \& S.G. MERRIFIELD. 1989. The physicochemistry, flora and fauna of intermittent prairie streams. A review of the literature. U.S. Fish and Wildlife Service. Biological report, 89 (5). 